DOI: $10.15593 / 24111678 / 2016.03 .01$

УДК 551.510 .42

\author{
Т.Г. Анодина', И.Ш. Магеррамов² \\ ${ }^{1}$ Межгосударственный авиационный комитет, Москва, Россия \\ ${ }^{2}$ Национальное аэрокосмическое агентство, \\ Баку, Азербайджанская Республика

\section{ВОПРОСЫ ПОСТРОЕНИЯ ЭКОЛОГИЧЕСКИХ ИЗМЕРИТЕЛЬНЫХ СЕТЕЙ КОНТРОЛЯ КАЧЕСТВА ВОЗДУХА В АЭРОПОРТАХ}

\begin{abstract}
До 1990-х гг. контроль воздействия на окружающую среду в аэропортах ограничивался измерением уровня шума, однако исследования последних лет убедительно доказали актуальность строгого наблюдения и за уровнем загрязнения атмосферы над аэропортами. Результаты изучения концентраций таких загрязнителей, как $\mathrm{CO}, \mathrm{NO}, \mathrm{NO}_{2}, \mathrm{PM}$, показали их четко выраженный импульсный характер, что подтверждает их взаимосвязь с режимами взлета и посадки самолетов. В последние годы были разработаны и внедрены различные измерительные комплексы экологического назначения для контроля за чистотой воздуха в аэропортах. Вместе с тем вопрос об информационной оптимизации структуры измерительных сетей не был поставлен и решен. Его особенность заключается в необходимости рассмотрения продвижения информационных потоков со всех измерительных станций в единый центр обработки данных с учетом ограничений на канал передачи сообщений. В данной статье сформулирована и решена задача оптимизации экологической измерительной сети в аэропортах, позволяющая оптимизировать структуру экологических измерительных сетей, устанавливаемых в зоне аэропорта. Согласно полученному решению в оптимальной измерительной сети количество используемых типовых блоков должно быть пропорционально количеству измерительных градаций, имеющихся в данном типе блока измерителя.
\end{abstract}

Ключевые слова: атмосфера, аэропорт, оптимизация, измерительные сети, качество воздуха, информация.

\author{
T.G. Anodina ${ }^{1}$, I.Sh. Magerramov ${ }^{2}$ \\ ${ }^{1}$ Intergovernmental Aviation Committee, Moscow, Russian Federation \\ ${ }^{2}$ National Aerospace Agency, Baku, Azerbaijan Republic
}

\title{
QUESTIONS ON DEVELOPMENT OF ECOLOGICAL MEASURING NETWORKS FOR CONTROL OF AIR QUALITY IN AIRPORTS
}

Till 1990th the control of impact on environment in airports was limited by control the level of noises generated by airplanes but held researches afterwards had shown the actuality of both the noise level and pollution of atmosphere over the airports. Results of measurements of concentrations of such polluters as $\mathrm{CO}, \mathrm{NO}, \mathrm{NO}_{2}, \mathrm{PM}$ had shown their pulse character and correlation with regime of landing and taking off procedures. Past years the various measuring complexes of ecological designation were developed and installed for control of air quality in airports. At the same time such important question as information optimization of measuring networks structure was not formulated and solved. The peculiarity of this question is that the movement of information flows from all measuring stations directed to proc- 
essing center should be taken into account jointly with limitation imposed on data transmission channel capacity. In the article the task of optimization of ecological measuring network in airports is formulated and solved. The formulated and solved optimization task make it possible to optimize the structure of ecological measuring networks, installed in airport zone. According to derived solution the number of used typical measuring blocks should be proportional to number of measuring gradations utilized in the this type of meter.

Keywords: atmosphere, airports, optimization, measuring networks, air quality, information.

Как отмечается в работе [1], те загрязнения воздуха, которые исходят от аэропортов, в особенности в момент прибытия и вылета самолетов, в настоящее время интенсивно исследуются. Не случайно, что во многих развитых странах эксплуатируются развитые системы контроля эмиссий в атмосферу в аэропортах [2-7].

До 1990-х гг. контроль воздействия на окружающую среду в аэропортах ограничивался измерением уровня шума, однако исследования последних лет убедительно доказали актуальность строгого наблюдения и за уровнем загрязнения атмосферы над аэропортами. Как отмечается в работах [8-13], загрязнение атмосферы над аэропортами происходит в основном при взлете и посадке самолетов, а также при выруливании лайнеров к полосе взлета и обратно к терминалу. В работе [1] изложены концептуальные основы применения системы мониторинга за окружающей средой в воздушном транспорте (REMSAT) в таких аэропортах, как Международный аэропорт Даллес (Вашингтон), а также Национальный аэропорт Рональд Рейган (Вашингтон).

Одной из основных особенностей экологического контроля в этих аэропортах является использование автоматических станций для наблюдений на земле (ASOS), ежечасные измерительные данные которых позволяют оценить не только метеорологическую обстановку, но и взаимосвязь между уровнем шума в аэропорту и степенью загрязнения воздуха. Измерительные станции расположены вокруг аэропортов с радиусом 12 морских миль, при этом используется как стационарный, так и мобильный принцип установки оборудования.

В работе [2] рассмотрены некоторые вопросы построения и эксплуатации станций аэрозольных измерений в лондонском аэропорту Хитроу (Англия). Согласно [2] в аэропорту Хитроу внедрена самая развитая система контроля качества в Европе. В 12 станциях, расположенных на территории аэропорта и в его окрестностях, измеряются концентрации таких загрязнителей, как $\mathrm{NO}, \mathrm{NO}_{2}, \mathrm{PM}_{10}, \mathrm{PM}_{2,5}, \mathrm{SO}_{2}, \mathrm{O}_{3}$.

Для измерения концентрации аэрозолей $\mathrm{PM}_{10}$ и $\mathrm{PM}_{2,5}$ используется аппаратура ТЕОМ - анализатор микрочастиц. Согласно данным, 
приведенным в работе [2], концентрация $\mathrm{PM}_{10}$ в зоне аэропорта на 10-15\% выше, чем в его окрестностях (рис. 1), однако не повышает

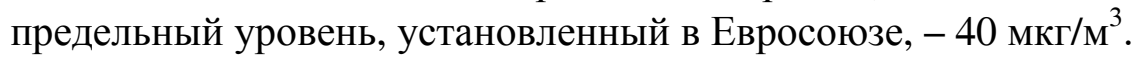

В вышеуказанной работе также сообщается об использовании в мониторинговых целях аппаратуры TSI Model 3010 - конденсационного счетчика частиц, позволяющего измерить диаметр частиц от 7 нм до 3 мкм.

Проведенные измерения пиковых значений $\mathrm{PM}_{2,5}$ и $\mathrm{PM}_{10}$ показали их хорошую взаимосвязь с режимом функционирования двигателей прибывающих и вылетающих самолетов. На рис. 2 приведены измеренные значения концентрации $\mathrm{NO}_{2}$ в аэропорту и в его окрестностях.

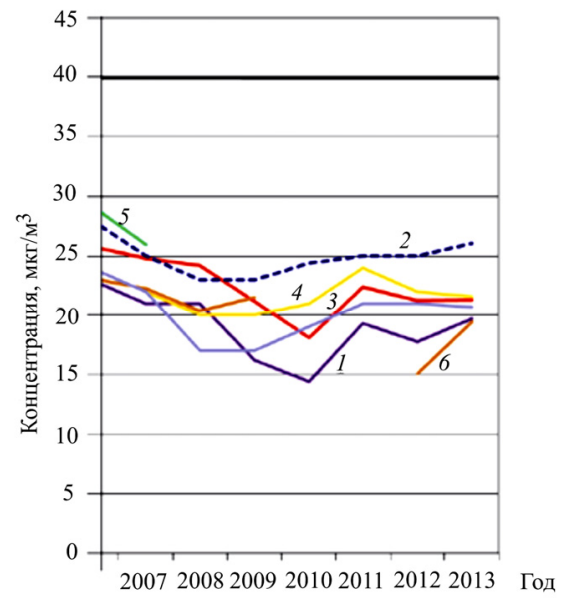

Рис. 1. Результаты измерения концентрации $\mathrm{PM}_{10}$ в аэропорту Хитроу и в его окрестностях [2]: 1 - Оксфордский проспект; 2 - Хитроу LHR2; 3 - Лондон Харлингтон; 4 - Зеленые ворота; 5 - Лондон Халингдон; 6 Кренфорд

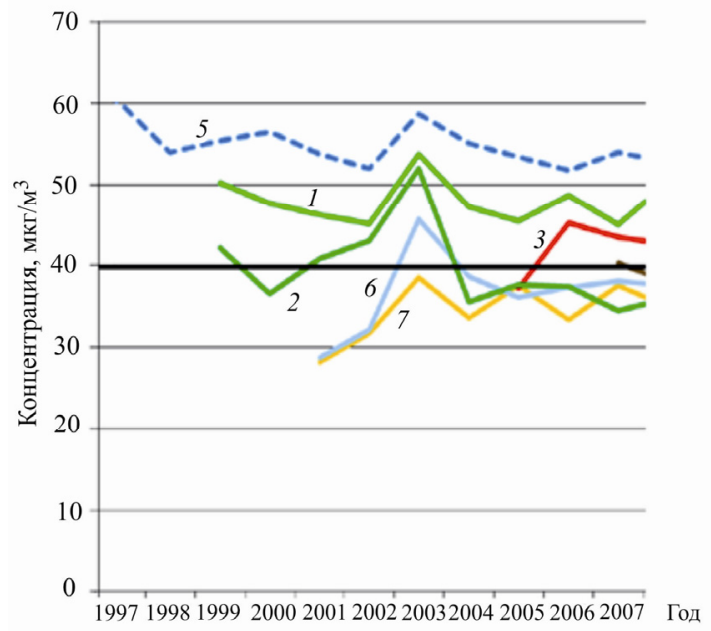

Рис. 2. Измеренные значения концентрации $\mathrm{NO}_{2}$ в аэропорту Хитроу и в его окрестностях [2]: 1 - Зеленые ворота; 2 - Хаттон Х; 3 - Харлингтон; 4 - Хармондсворд; 5 - Хитpoy LHR2; 6 - Кренфорд; 7 - Оксфордский проспект

Из приведенных графиков видно, что концентрация $\mathrm{NO}_{2}$ в аэропорту существенно превышала предельный уровень 40 мкг/м ${ }^{3}$, установленный в Евросоюзе. При этом, как видно из графиков, представленных на рис. 3, измеренные значения концентрации таких соединений, как $\mathrm{CO}, \mathrm{NO}, \mathrm{NO}_{2}, \mathrm{PM}$, носят четко выраженный импульсный характер [3], что подтверждает их взаимосвязь с режимами взлета 

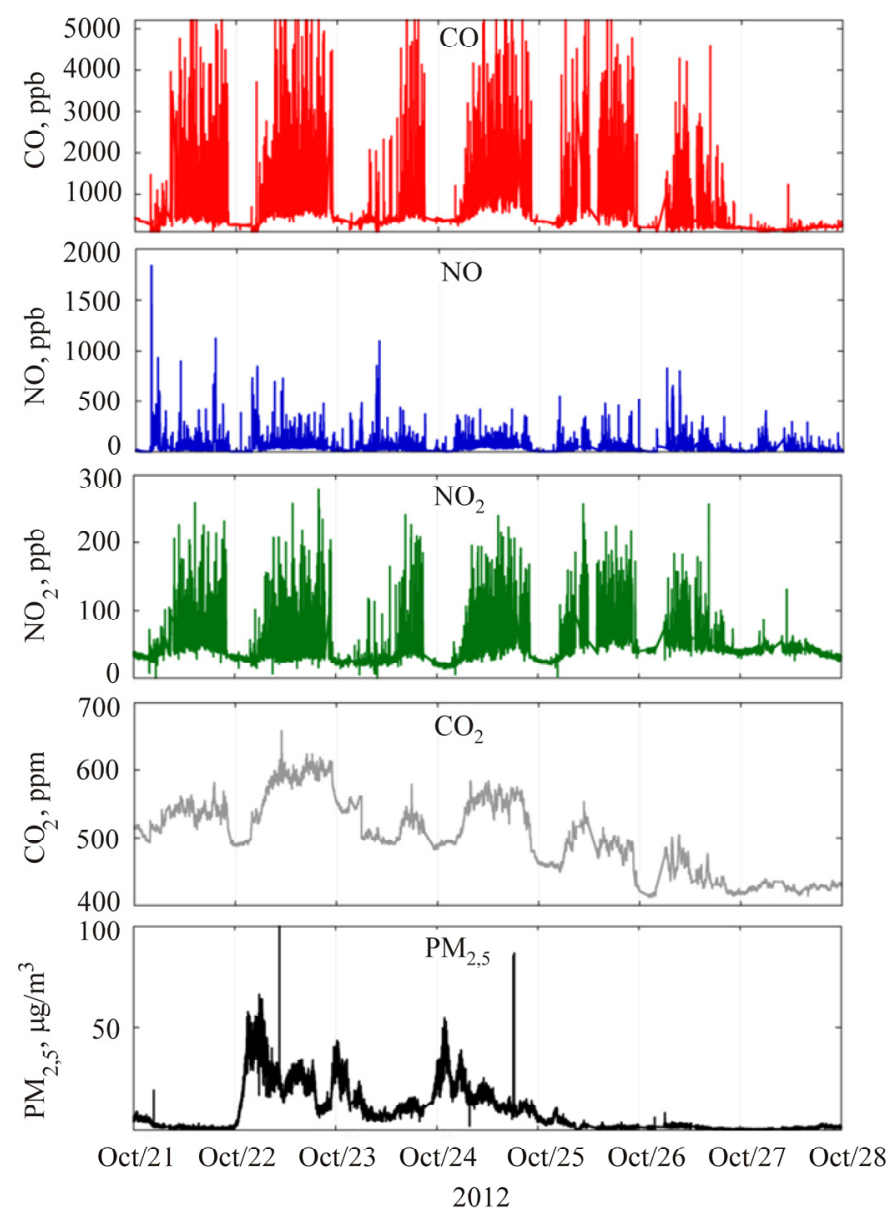

Рис. 3. Результаты измерений концентрации СO, NO, $\mathrm{NO}_{2}, \mathrm{CO}_{2}, \mathrm{PM}_{2,5}$ в аэропорту Хитроу [3]

и посадки самолетов. Аналогичные результаты также были получены в работах $[15,16]$.

В работе [4] рассмотрены некоторые вопросы оптимизации режима функционирования вышеизложенных экологических измерительных сетей в зоне аэропорта и в его окрестностях. Вместе с тем важный вопрос об информационной оптимизации структуры измерительных сетей не был поставлен и решен. Его особенность заключается в необходимости рассмотрения продвижения информационных потоков со всех измерительных станций в единый центр обработки данных с учетом ограничений на канал передачи сообщений. При этом в качестве дистанционных измерителей могут быть использованы спектрометрические и лидарные устройства [14, 18], а в центре обработки - 
искусственные нейронные сети [17]. Ниже сформулирована задача оптимизации экологической измерительной сети в аэропортах, а также представлено ее решение.

Допускается, что измерительные станции, размещаемые в аэропорту и в его окрестностях, имеют блочную структуру и могут содержать некоторые из следующих блоков:

- блок измерения концентрации СО;

- блок измерения концентрации $\mathrm{NO}$;

- блок измерения концентрации $\mathrm{NO}_{2}$;

- блок измерения концентрации $\mathrm{CO}_{2}$;

- блок измерения концентрации $\mathrm{PM}_{2,5}$;

- блок измерения концентрации $\mathrm{PM}_{10}$.

При этом максимальное количество блоков в станции в рассматриваемом случае равно 6, но на практике может быть и больше. Допускаем, что каждый тип блоков измерения имеет свое фиксированное количество измеряемых градаций $P_{i}$. Если в сети используется $N_{i}$ число блоков типа $i$, то общее количество информации, вырабатываемой всеми блоками типа $i$, может быть оценено как

$$
M_{i}=N_{i} \log _{2} P_{i} .
$$

Количество информации, вырабатываемое всеми типами измерительных блоков, определим как

$$
M_{\Sigma}=\sum_{i=1}^{n} N_{i} \log _{2} P_{i}
$$

где $n$ - количество типов блоков.

Формируемая и решаемая оптимизационная задача представляется следующим образом. Вводится на рассмотрение функция

$$
P_{i}=\xi\left(N_{i}\right)
$$

определяющая зависимость количества измерительных градаций от количества блоков измерений рассматриваемого типа.

С учетом выражений (2) и (3) получим

$$
M_{\Sigma}=\sum_{i=1}^{n} N_{i} \log _{2} \xi\left(N_{i}\right)
$$

Ограничение на трафик измерительной информации задается в следующем виде: 


$$
\sum_{i=1}^{n} \xi\left(N_{i}\right)=C_{1}
$$

где $C_{1}=$ const.

В частном случае условие (5) может быть интерпретировано в бинарном виде (рис. 4), где согласно ему площадь треугольника $O B D$ должна быть равна площади треугольника $O C D$.

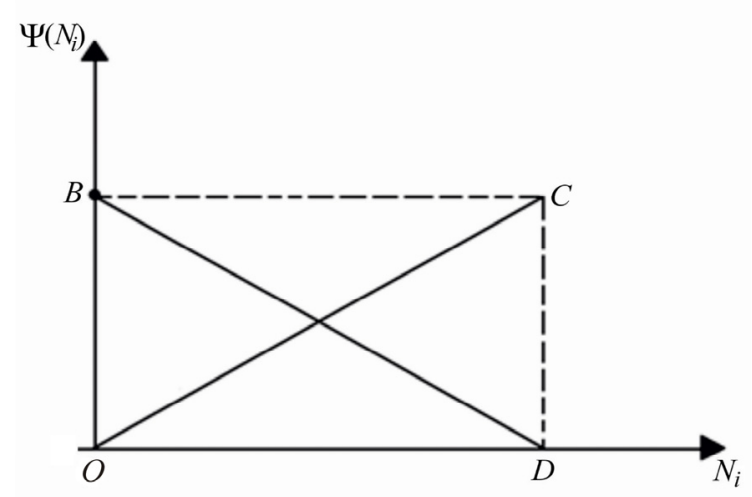

Рис. 4. Бинарная интерпретация условия (5)

С учетом выражений (4) и (5) можно сформировать следующий дискретный функционал безусловной вариационной оптимизации:

$$
F_{g}=\sum_{i=1}^{n} N_{i} \log _{2} \xi\left(N_{i}\right)+\chi \sum_{i=1}^{n} \xi\left(N_{i}\right)
$$

где $\chi$ - множитель Лагранжа.

Дискретный функционал (6) может быть представлен в непрерывном виде

$$
F_{H}=\int_{0}^{N_{\max }} N \cdot \log _{2} \xi(N)+\chi \int_{0}^{N_{\max }} \xi(N) d N
$$

при

$$
\int_{0}^{N_{\max }} \xi(N) d N=C_{2}
$$

где $C_{2}=$ const.

Для нахождения оптимального вида функции $\psi(N)_{\text {opt }}$ воспользуемся методом Эйлера, согласно которому функция $\psi(N)_{\text {opt }}$ должна удовлетворить условию 


$$
S_{1}=\frac{d\left\{N \log _{2} \xi(N)+\chi \xi(N)\right\}}{d \xi(N)}=0 .
$$

Из условия (8) получим

$$
\frac{N}{(\ln 2) \cdot \xi(N)}+\chi=0
$$

Из выражения (10) находим

$$
\xi(N)=-\frac{N}{\chi \cdot \ln 2} .
$$

Учитывая условие (11) в формуле (8), получаем

$$
-\int_{0}^{N_{\max }} \frac{N}{\chi \cdot \ln 2} d N=C_{2} .
$$

Из выражения (12) находим

$$
\chi=-\frac{N_{m}^{2}}{2 C_{2} \cdot \ln 2} .
$$

С учетом выражений (10) и (13) окончательно находим

$$
\xi(N)=\frac{2 C_{2} \cdot N}{N_{m}^{2}}
$$

Таким образом, при обеспечении функциональной зависимости (14) информативность сети может достичь экстремальной величины. Для определения типа экстремума достаточно вычислить выражение

$$
S_{1}=\frac{d^{2}\left\{N \cdot \log _{2} \xi(N)+\chi \xi(N)\right\}}{d \xi(N)^{2}}=0
$$

и убедиться, что $S_{2}$ при условии (14) достигает отрицательной величины, т.е. выражение (14) обеспечивает максимум функционала (7).

Таким образом, сформулированная и решенная оптимизационная задача позволяет оптимизировать структуру экологических измерительных сетей, устанавливаемых в зоне аэропорта. Согласно полученному решению в оптимальной измерительной сети количество используемых типовых блоков должно быть пропорционально количеству измерительных градаций, имеющихся в данном типе блока измерителя. 


\section{Список литературы}

1. Regional environmental monitoring system for air traffic / L. Azahari, J. Duenas, W.J. Hamilton, J. Hanifin // 2009 IEEE Systems and Information Engineering Design Symposium. - Sieds, 2009. - P. 246-251. DOI: 10.1109/SIEDS.2009.5166189

2. Vowles D. Heathrow airport particulate measurements 10th January 2014. - URL: http://www.forum-ae.eu/system/files/00_forum-ae_ workshop_9-10_january_2014_version_8jan14.pdf (дата обращения: 01.09.2016).

3. Popoola L. Application of a high density low cost sensor network for air quality (SNAQ) at London Heathrow Airport. - URL: http://www.ch.cam.ac.uk/files/ posters/Jones(Rod)1.pdf (дата обращения: 01.09.2016).

4. Characteristics of ozone concentrations at stations with different backgrounds in the Malaysian Peninsula / N. Banan, M.T. Latif, L. Juneng, F. Ahamad // Aerosol and Air Quality Research. - 2013. - Vol. 13. P. 1090-1106.

5. Measuring air pollutants in an international Romania airport with point and open path instruments / I. Ionel, D. Nicolae, F. Popescu C. Talianu, J. Belegante, G. Apostol // Romanian Journal of Physics. - 2011. Vol. 56, № 3-4. - P. 507-519.

6. Daley P.S., Naugle D.F. Measurement and analysis of airport emissions // Journal of the Air Pollution Control Association. - 1979. Vol. 29, № 2. - P. 113-116. DOI: 10.1080/00022470.1979.10470763

7. Wang Y.W., Yang J., Jiang W.M. Impact of large cities on air pollution // International Workshop on Geoscience and Remote Sensing: proceedings. - 2008. - Vol. 1. - P. 393-396.

8. Aviation emissions, impacts and mitigation a primer. - URL: http://www.faa.gov/regulations_policies/plicy_guidancr/enver_plicy/media/ Primer_Jan 2015.pdf (дата обращения: 01.09.2016).

9. Travis M.N. Aircraft greenhause gas emissions during the landing and take if cycle at bay area airports. - URL: http://repository. usca.edu/capstone (дата обращения: 01.09.2016).

10. IPCC good practice guidance and uncertainty management in national greenhouse gas inventories. Ch. 2. Energy. - URL: http://www. ipcc-nggip.iges.or.jp/public/gp/bgp/2_5_Aircraft.pdf (дата обращения: 01.09.2016). 
11. Aircraft and airport-related hazardous air pollutants: research needs and analysis / E. Wood, S. Herndon, R. Miake-Lye, D. Nelson // ACRP Report 7. Transportation Research Board. - Washington DC, 2008. 56 p. - URL: https://www.nap.edu/read/14168/ chapter/1 (дата обращения: 01.09.2016).

12. Aircraft taxiing strategy optimization / M.I.M.D. Ithnan, T. Selderbeek, W.W.A. Beelaerts van Blokland, G. Lodewijks. - URL: http://rstail.nl/new/wp-content/uploads/2015/02/izzudin_ithnan.pdf (дата обращения: 01.09.2016).

13. Aircraft impacts on local and regional air quality in the United States: partnership for air transportation noise and emissions reduction project 15 final report / G. Ratliff, Ch. Sequeira, I. Waitz, M. Ohsfeldt, T. Thrasher, M. Graham, T. Thompson. - 2009. - 181 p.

14. Nicolae D., Cristescu C.P. Laser remote sensing of tropospheric aerosol // Journal Optoelectronic Advance Materials. - 2006. - Vol. 8, № 5. - P. 1781-1795.

15. Popp P.J., Bishop G.A., Stedman D.H. Method for commercial aircraft nitric oxide emission measurements // Environmental Science and Technology. - 1999. - Vol. 33, № 3. - P. 1542-1552.

16. $\mathrm{NO}$ and $\mathrm{NO}_{2}$ emission ratios measured from in-use commercial aircraft during taxi and takeoff / C.H.N. Scot, H.S. Joanne, D.N. David, J. John, C.B. Robert, C. Richard, W. Ian, S. Phillip, L. Thomas, D. Ken and E.K. Charles Z. Marks // Environmental Science and Technology. - 2004. Vol. 38. - P. 6078-6084.

17. Gardner M.W., Dorling S.R. Artificial neural networks (the multilayer perceptron): a review of applications in the atmospheric sciences // Atmospheric Environment. - 1998. - Vol. 32. - P. 2627-2636.

18. High spectral resolution ozone absorption cross-section. Part 1. Measurements, data analysis and comparison with previous measurements around 293 K / V. Gorshelev, A. Serdyuchenko, M. Weber, W. Chehade, J.P. Burrows // Atmospheric Measurement Techniques. - 2014. - Vol. 7. P. 609-624.

\section{References}

1. Azahari L., Duenas J., Hamilton W.J., Hanifin J. Regional environmental monitoring system for air traffic. 2009 IEEE Systems and Infor- 
mation Engineering Design Symposium. Sieds, 2009, pp. 246-251. DOI: $10.1109 /$ SIEDS.2009.5166189

2. Vowles D. Heathrow airport particulate measurements 10th January 2014, available at: http://www.forum-ae.eu/system/files/00_forumae_workshop_9-10_january_2014_version_8jan14.pdf (accessed 01 September 2016).

3. Popoola L. Application of a high density low cost sensor network for air quality (SNAQ) at London Heathrow Airport, available at: http://www.ch.cam.ac.uk/files/posters/Jones(Rod)1.pdf (accessed 01 September 2016).

4. Banan N., Latif M.T., Juneng L., Ahamad F. Characteristics of ozone concentrations at stations with different backgrounds in the Malaysian Peninsula. Aerosol and Air Quality Research, 2013, vol. 13, pp. 10901106.

5. Ionel I., Nicolae D., Popescu F. Talianu C., Belegante J., Apostol G. Measuring air pollutants in an international Romania airport with point and open path instruments. Romanian Journal of Physics, 2011, vol. 56, no. 3-4, pp. 507-519.

6. Daley P.S., Naugle D.F. Measurement and analysis of airport emissions. Journal of the Air Pollution Control Association, 1979, vol. 29, no. 2, pp. 113-116. DOI: 10.1080/00022470.1979.10470763

7. Wang Y.W., Yang J., Jiang W.M. Impact of large cities on air pollution. Proceedings "International Workshop on Geoscience and Remote Sensing”, 2008, vol. 1, pp. 393-396.

8. Aviation emissions, impacts and mitigation a primer, available at: http://www.faa.gov/regulations_policies/plicy_guidancr/enver_plicy/media/ Primer_Jan2015.pdf (accessed 01 September 2016).

9. Travis M.N. Aircraft greenhause gas emissions during the landing and take if cycle at bay area airports, available at: http://repository. usca.edu/capstone (accessed 01 September 2016).

10. IPCC good practice guidance and uncertainty management in national greenhouse gas inventories. Ch. 2. Energy, available at: http://www.ipcc-nggip.iges.or.jp/public/gp/bgp/2_5_Aircraft.pdf (accessed 01 September 2016).

11. Wood E., Herndon S., Miake-Lye R., Nelson D. Aircraft and airport-related hazardous air pollutants: research needs and analysis. ACRP Report 7. Transportation Research Board. Washington DC, 2008. 56 p., 
available at: https://www.nap.edu/read/14168/chapter/1 (accessed 01 September 2016).

12. Ithnan M.I.M.D., Selderbeek T., Beelaerts van Blokland W.W.A., Lodewijks G. Aircraft taxiing strategy optimization, available at: http://rstail.nl/new/wp-content/uploads/2015/02/izzudin_ithnan.pdf (accessed 01 September 2016).

13. Ratliff G., Sequeira Ch., Waitz I., Ohsfeldt M., Thrasher T., Graham M., Thompson T. Aircraft impacts on local and regional air quality in the United States. 2009. 181 p.

14. Nicolae D., Cristescu C.P. Laser remote sensing of tropospheric aerosol. Journal Optoelectronic Advance Materials, 2006, vol. 8, no. 5, pp. 1781-1795.

15. Popp P.J., Bishop G.A., Stedman D.H. Method for commercial aircraft nitric oxide emission measurements. Environmental Science and Technology, 1999, vol. 33, no. 3, pp. 1542-1552.

16. Scot C.H.N., Joanne H.S., David D.N., John J., Robert C.B., Richard C., Ian W., Phillip S., Thomas L., Ken D., Charles E.K., Marks Z. $\mathrm{NO}$ and $\mathrm{NO}_{2}$ emission ratios measured from in-use commercial aircraft during taxi and takeoff. Environmental Science and Technology, 2004, vol. 38, pp. 6078-6084.

17. Gardner M.W., Dorling S.R. Artificial neural networks (the multilayer perceptron). Atmospheric Environment, 1998, vol. 32, pp. 2627-2636.

18. Gorshelev V., Serdyuchenko A., Weber M., Chehade W., Burrows J.P. High spectral resolution ozone absorption cross-section. Part 1. Measurements, data analysis and comparison with previous measurements around 293 K. Atmospheric Measurement Techniques, 2014, vol. 7, pp. 609-624.

Получено 14.09.2016

\section{Об авторах}

Анодина Татьяна Григорьевна (Москва, Российская Федерация) - доктор технических наук, профессор, председатель Межгосударственного авиационного комитета (19017, Москва, ул. Большая Ордынка, 22/2/1, e-mail: mak@mak.ru).

Магеррамов Ибрагим Шаиг оглы (Баку, Азербайджанская Республика) - аспирант, Национальное аэрокосмическое агентство 
(AZ1106, Азербайджанская Республика, Баку, ул. С.С. Ахундова, 1, imaharramov@hotmail.com).

\section{About the authors}

Tat'iana G. Anodina (Moscow, Russian Federation) - Doctor of Technical Sciences, Professor, Chairman of Intergovernmental Aviation Committee (22/2/1, Bolshaya Ordynka st., Moscow, 119017, Russian Federation, e-mail:mak@mak.ru).

Ibragim Shaig ogly Magerramov (Azerbaijan Republic, Baku) Postgraduate Student, National Aerospace Agency (1, S.S. Akhundov st., Baku, AZ1106, Azerbaijan Republic, e-mail: imaharramov@ hotmail.com). 